\title{
Bosques oligárquicos de Oecopetalum mexicanum enriquecidos con especies nativas de la Sierra de Misantla, México
}

\section{Oligarchic forests of Oecopetalum mexicanum enriched with native species of Sierra de Misantla, Veracruz, Mexico}

\author{
Melissa Covarrubias', Juan Carlos López-Acosta², Maite Lascurain-Range $3^{3}$, \\ Virginia Rebolledo ${ }^{4}$, Rosa Amelia Pedraza ${ }^{4}$ y Sergio Avendaño-Reyes ${ }^{5}$
}

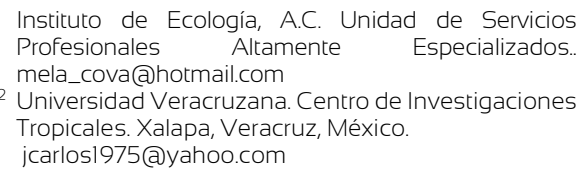

2 Universidad Veracruzana. Centro de Investigaciones Tropicales. Xalapa, Veracruz, México. jcarlos1975@yahoo.com

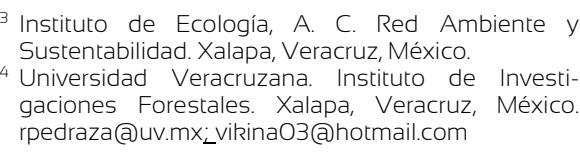
Sustentabilidad. Xalapa, Veracruz, México.

${ }_{4}$ Universidad Veracruzana. Instituto de Investigaciones Forestales. Xalapa, Veracruz, México rpedraza@uv.mxivikinaO3@hotmail.com

\author{
5 Instituto de Ecología, A. C. Herbario XAL. \\ sergio.avendano@inecol.mx \\ * Autor de correspondencia. maite.lascurain@inecol
}

\section{RESUMEN}

El enriquecimiento de los bosques manejados es una práctica que implica la inserción de especies de valor cultural, económico y ecológico. Son sistemas que han sido históricamente modificados y manejados por el hombre para favorecer una sola especie. Ejemplo de ello son los cachichinales, bosquecillos típicos de la región Sierra de Misantla, en el centro de Veracruz, dominados por Oecopetalum mexicanum, un árbol de semillas comestibles. En este estudio se probaron dos técnicas de plantación para su enriquecimiento: translocación y trasplante de vivero. Se utilizaron tres especies arbóreas altamente valoradas en la región: Ocotea puberula, Beilschmiedia anay y Pseudolmedia glabrata. Para evaluar el estado fisiológico, crecimiento y supervivencia de las plantas se utilizó un análisis de varianza. Las tres especies tuvieron un marcado estrés (estado de la planta) en las primeras etapas de establecimiento. Después de 12 meses se registró una supervivencia mayor a 50\%, indistintamente entre las técnicas utilizadas para cada especie. El crecimiento de O. puberula fue mayor (promedio y probabilidad) en el ensayo de translocación, en comparación con las otras especies. Ambas técnicas de siembra tuvieron una alta supervivencia, por lo que es factible utilizarlas indistintamente en proyectos de introducción de especies nativas en bosques enriquecidos.

Palabras Clave: Beilschmiedia anay, Ocotea puberula, Pseudolmedia glabrata, supervivencia, tasa de crecimiento, manejo forestal.

\begin{abstract}
The practice of enrichment of managed forests implies the insertion of species with cultural, economic and ecological value. It generally takes place in sites with some degree of anthropic impact, and also applies in sites known as oligarchic forests. They are historically modified and man-managed systems to favor a single species. Examples are the cachichinales woodlands typical of the Sierra de Misantla region, Veracruz, dominated by Oecopetalum mexicanum, a tree of edible seeds. In this study, two planting techniques were tested: translocation and nursery transplantation of three tree species highly valued in the region: Ocotea puberula, Beilschmiedia anay and Pseudolmedia glabrata. We used a paired model (Anova) to evaluate its physiological state, growth and survival. All three species showed marked stress in the early stages of their establishment; a survival rate greater than $50 \%$ was recorded after one year, regardless the technique used for either specie. Growth-wise, O. puberula was the most successful; although we found overall high survivals rates in all three species, which lead us to conclude it is feasible to introduce them into cachichinales.
\end{abstract}

KEYWORDS: Beilschmiedia anay, Ocotea puberula, Psendolmedia glabrata, survival, growt rate, forest management. 


\section{INTRODUCCIÓN}

El término enriquecimiento de bosques se refiere a la inserción selectiva de especies con características ecológicas, culturales y económicas relevantes para los productores, en espacios con distintos grados de perturbación (Montagnini, Eibl, Grance, Maiocco, y Nozzi 1997). Esta práctica es usualmente aplicada a escenarios donde hay especies localmente valiosas que pueden ser insertadas en sitios con baja riqueza biológica. El enriquecimiento de bosques tiene varias ventajas: i) facilita la recolección de las especies de interés; ii) reduce la distancia para la recolecta; iii) disminuye la presión sobre las poblaciones naturales; iv) aumenta el valor de mantenimiento y empoderamiento de los bosques; v) disminuye la amenaza de conversión a otros usos de la tierra y, en consecuencia, ayuda a conservar estas asociaciones vegetales productivas (López-Acosta, Lascurain, LópezBinnqüist y Covarrubias, 2014) y vi) es una técnica frecuentemente utilizada en la restauración de bosques. Estos sistemas pueden servir de resguardo de especies relevantes para el funcionamiento ecológico y la subsistencia de las personas (Mansourian, Lamb y Gilmour, 2005; Ticktin, Johns y Xoca, 2003).

Generalmente las prácticas de enriquecimiento están dirigidas a bosques de bajo o alto impacto antrópico (Aide, Zimmerman, Pascarella, Rivera y Marcano-Vega, 2000; Martínez-Garza y Howe 2003; Paquette, Léonard, Lundgren-Cayrol, Mihaila y Gareau, 2006). No obstante, existen otros sistemas que han sido modificados y manejados por el hombre por largos periodos, denominados oligárquicos, monotípicos o enriquecidos (Schroth et al., 2004). Este tipo de bosques están configurados a través de un patrón silvícola perenne que integra paulatinamente especies relevantes de la matriz forestal, lo cual los hace "invisibles" para el observador convencional, pues poseen una estructura y composición vegetal similares a los bosques, pero que no corresponde a una clasificación de vegetación específica (Michon, 2005; Peters, 2000).

Los bosques oligárquicos se caracterizan también por contener, en abundancia, especies pivote que son clave para la subsistencia de los habitantes locales (Mansourian et al., 2005); normalmente son de árboles nativos, los cuales muchas veces son conocidos por su nombre local, por ejemplo: los "azaisales" en Brasil, que son sistemas donde predomina la palma Euterpe oleracea Mart. (açai); en el sur de México los "ramonales" dominados por Brosimun allicastrum Sw. (ramón u ojoche) y; en el centro del estado de Veracruz los "cachichinales", dominados por Oecopetalum mexicanum Greenm. \& C. H. Thomps. (cachichín). En todos estos sistemas los campesinos cuidan los árboles valiosos, sean originarios del bosque o del cultivo circundante, eliminan las especies menos deseadas y con ello incrementa su valor económico y cultural.

Los cachichinales son pequeños bosquecillos (con superficie de un cuarto a una hectárea) típicos de la sierra de Misantla, en la región central del estado de Veracruz (López-Acosta et al., 2014; Lascurain et al., 2013; Covarrubias, 2010). Los árboles de Oecopetalum mexicanum producen una semilla comestible que forma parte de la identidad regional (Lascurain, López-Binnqüist y Emery, 2016). Estos sistemas presentan características estructurales y fisonómicas similares a la vegetación original del bosque mesófilo de montaña, la selva alta perennifolia y sus ecotonos (Gómez-Pompa, 1966; Gutiérrez, 1993).

Los cachichinales, como muchos de los bosques enriquecidos del país, no aparecen en ningún tipo de clasificación de uso de suelo que facilite el desarrollo de políticas públicas y/o estrategias para su conservación y mantenimiento, lo cual los pone aún más susceptibles que los ecosistemas naturales. Por tal motivo, es necesario desarrollar estrategias dirigidas a maximizar su uso, enriqueciéndolos mediante la introducción de especies probadamente útiles a escala local, que aumenten su valor comercial y de subsistencia. De esta manera su mantenimiento e inserción puedan ser justificados en las políticas públicas con el fin de asegurar su aprovechamiento y manejo.

\section{OBJETIVOS}

El objetivo de este trabajo fue evaluar tres especies de árboles útiles y dos técnicas de manejo para el 
enriquecimiento de los cachichinales: la translocación de plantas silvestres y el trasplante de plantas cultivadas en vivero. Estos ensayos son de gran importancia en estrategias de manejo forestal y conservación de la diversidad y pueden ser replicados en diferentes regiones del país, según el contexto ecológico.

\section{MATERIALES Y MÉTODOS}

\section{Área de estudio}

Los bosques oligárquicos dominados por Oecopetalum mexicanum se encuentran ubicados en el centro del estado de Veracruz, en la denominada sierra de Misantla que deriva de la Sierra Madre Oriental y el Eje Neovolcánico Transversal. El área de estudio pertenece al municipio de Misantla, localizado entre las coordenadas $19^{\circ} 56^{\prime}$ latitud norte y $96^{\circ}$ 51' longitud oeste, a una altura de $300 \mathrm{~m}$ snm (Fig. 1). Sus suelos son de tipo luvisol y vertisol. El clima para esta zona es cálido húmedo Af (m) con lluvias todo el año, en donde domina la selva alta subperennifolia como sistema original en una matriz agrícola.

\section{Especies para ensayo de enriquecimiento}

Se eligieron tres especies de árboles cultural y económicamente relevantes en la región: Beilschmiedia anay (S.F. Blake) Kosterm. (anayo, escalán), Ocotea puberula (Rich.) Nees (alamanca) y Pseudolmedia glabrata (Liebm.) C.C. Berg (tepetomate).

\section{Beilschmiedia anay}

Árbol de hasta $22 \mathrm{~m}$ de altura y $70 \mathrm{~cm}$ de diámetro a 1.30 $\mathrm{m}$ de altura, respecto al suelo (DAP), tronco erguido, ramas ascendentes y copa densa. Corteza externa escamosa, morena rojiza. Hojas simples, generalmente dispuestas en espiral, de $13 \mathrm{~cm} \times 7 \mathrm{~cm}$ a $28 \mathrm{~cm} \times 13 \mathrm{~cm}$; verde brillante en el haz y pubescente en el envés; con olor fuerte a aguacate cuando se estrujan. Su nombre común en la región de estudio es escalán. Es un árbol originario de las regiones tropicales húmedas de América (Niembro-Rocas, Morato y Cuevas-Sánchez, 2004). Crece especialmente en laderas montañosas con suelos bien drenados y de origen volcánico o calizo (Pennington y Sarukhán, 2005), de 440 m a 700 m snm. El fruto es comestible y muy apreciado en el municipio

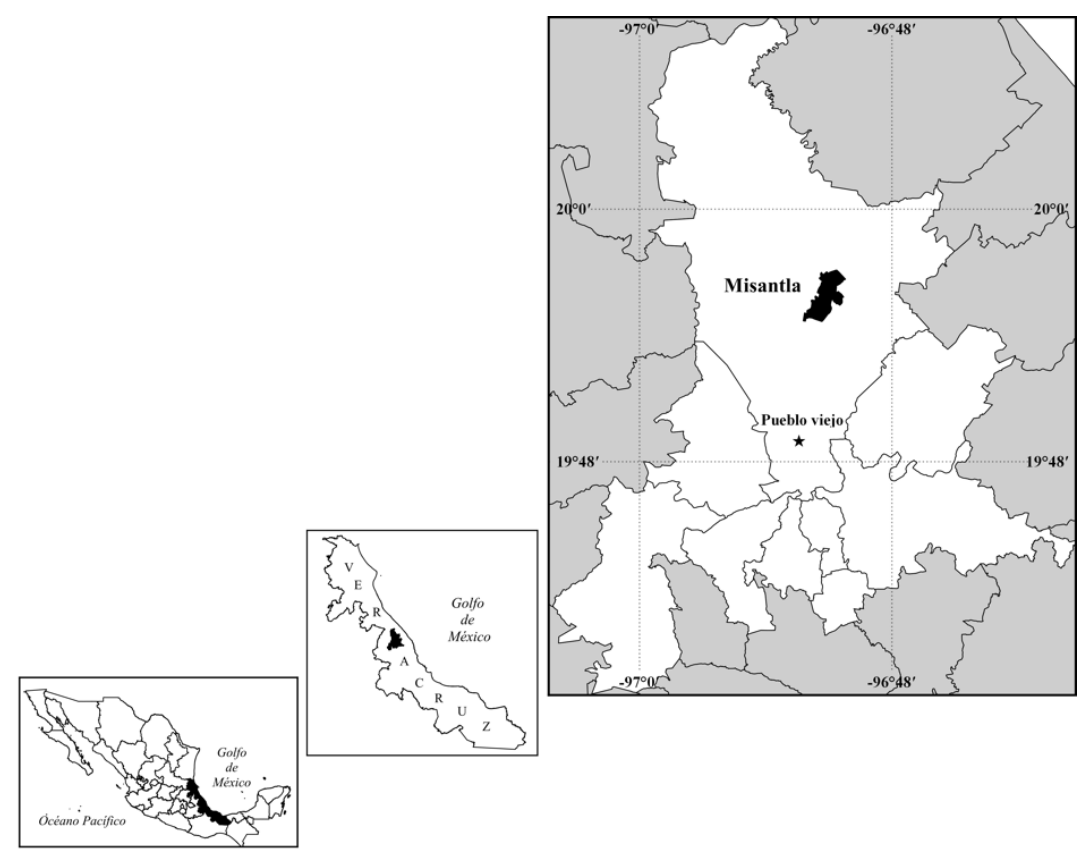

FIGURA 1. Mapa de localización del área de estudio en el municipio de Misantla, los ensayos se realizaron en la localidad de Pueblo Viejo, Municipio de Misantla, Veracruz, México. 
de Misantla. La madera es utilizada en la construcción, para leña, carbón, estacas, postes y elaboración de mangos para herramientas agrícolas. Niembro-Rocas et al. (2004) lo describen como un árbol raro, escasamente estudiado y apto para propagar dentro de programas de reforestación y restauración.

\section{Ocotea puberula}

Árbol de $7 \mathrm{~m}$ a $27 \mathrm{~m}$ de altura y hasta $70 \mathrm{~cm}$ de DAP, corteza café (joven) a gris pálido (adulta). Frutos de $6 \mathrm{~mm}$ a $8 \mathrm{~mm}$ de diámetro, globosos, color verde oscuro lustroso. Florece en el mes de noviembre y fructifica entre enero y febrero. Se distribuye en México (150 m a $1600 \mathrm{~m}$ snm), Centro y Sudamérica tropical, en clima cálido húmedo. Su hábitat son bosques bien conservados pero es una especie conspicua en áreas degradadas pues es dejada como remanente de los bosques originales. Su madera es usada para construcción de casas, cimbra, puertas, ventanas y muebles como camas y roperos por lo que es cultivada o simplemente se deja crecer en los terrenos para su aprovechamiento (Gutiérrez y Dorantes, 2007). Su nombre común en la sierra de Misantla es alamanca.

\section{Pseudolmedia glabrata}

Árbol de hasta $30 \mathrm{~m}$ de alto y hasta $1 \mathrm{~m}$ de DAP; tronco erguido, copa redonda y densa. Corteza externa gris parduzca, lisa a ligeramente escamosa en pequeños pedazos. Hojas alternas simples, de $5 \mathrm{~cm} \times 1.8 \mathrm{~cm}$ a $15 \mathrm{~cm}$ $\times 4.5 \mathrm{~cm}$, estrechamente elípticas u oblongas; verde amarillento a oscuro brillante en el haz y verde grisáceo en el envés. Flores masculinas en cabezuelas verdosas, aplanadas, axilares, solitarias de $1 \mathrm{~cm}$ de diámetro; flores femeninas solitarias o agrupadas en axilas de las hojas, de 3 $\mathrm{mm}$ a $6 \mathrm{~mm}$ de largo incluyendo el estilo. Florece de enero a abril y fructifica en abril y mayo (Lascurain et al., 2013). Los frutos son bayas de $1.5 \mathrm{~cm}$ a $2 \mathrm{~cm}$ de largo, ovoides o elipsoides, rodeadas completamente por el perianto acrescente rojo. Forma parte de selvas altas o medianas, perennifolias y subperennifolias, de $0 \mathrm{~m}$ a $700 \mathrm{~m} \mathrm{snm}$. El fruto es comestible, conocido en la región como tepetomate. La madera es muy apreciada localmente y es empleada para construcciones rurales, así como para elaborar mangos de herramientas agrícolas, también es apropiada para la fabricación de durmientes (Pennington y Sarukhán, 2005).

\section{Técnicas experimentales}

\section{Translocación}

Se trata del movimiento de organismos (en este caso plantas) de una localización a otra, con el fin de aumentar o introducir el número de especies con un objetivo particular en el sitio deseado. En este estudio se utilizaron 270 brinzales (90 de cada especie) que fueron tomados directamente del bosque y translocados hacia los cachichinales. Se hizo énfasis en que el tamaño de las plantas y la edad fuera homogéneo entre los individuos de cada especie: Beilschmiedia anay $27 \mathrm{~cm}-32 \mathrm{~cm}$ de altura, Ocotea puberula $13 \mathrm{~cm}-20 \mathrm{~cm}$ y Pseudolmedia glabrata $10 \mathrm{~cm}$ - $16 \mathrm{~cm}$. Asimismo, se procuró que tuvieran al menos 2 hojas verdaderas. Cada individuo fue extraído junto con un núcleo de tierra de al menos $20 \mathrm{~cm}^{2}$ para evitar el corte de raíces, y luego fue envuelto con papel periódico húmedo, esto con la finalidad de prevenir la desecación y daño en el traslado.

\section{Producción en vivero}

Las plantas fueron obtenidas de viveros rústicos de la localidad de Pueblo Viejo. Se utilizaron 270 brinzales sanos (90 de cada especie), provenientes de semillas del bosque, recolectadas de al menos 15 árboles parentales. Una vez alcanzado el tamaño del contingente de translocación (el cual proviene de la misma cohorte desde invernadero), las plantas fueron sembradas en los cachichinales.

\section{Ensayo de enriquecimiento}

El ensayo de enriquecimiento fue de 540 plantas (270 por técnica), las cuales fueron distribuidas y sembradas en tres diferentes cachichinales (180 plántulas por sitio). Con la finalidad de identificar las diferencias en supervivencia y crecimiento, según la técnica experimental, las plantas fueron dispuestas en un diseño por pares, con $50 \mathrm{~cm}$ de separación entre sí y conservando la misma altura con 
respecto a la pendiente; es decir, dos plantas de la misma especie, introducidas a campo en la misma fecha pero cada una mediante una técnica distinta, creciendo bajo las mismas condiciones ambientales, de cobertura, tipo de suelo y pendiente (Fig. 2).

La plantación se hizo en el periodo de lluvias (junio), se cuantificó la supervivencia de cada plántula mediante un monitoreo mensual (agosto 2012-agosto 2013). Según el estado fisiológico de cada plántula, se elaboró la siguiente categoría: viva $(\mathrm{V})$, muerta (M) y estresada (E). Esta última categoría fue definida como la pérdida de turgencia o etiolación de las hojas, aun cuando la planta presentara un tallo verde. Cada dos meses, se midió la altura de las plantas desde la base del tallo hasta el meristemo apical. Cada tres meses se registró el diámetro del tallo, por encima de la corona.

Se hicieron análisis descriptivos de todas las variables y se realizó un análisis de varianza factorial (Anova), se tomaron como factores la técnica (translocación $v s$. vivero) y cada una de las especies (Beilschmiedia anay, Ocotea puberula y Pseudolmedia glabrata) para determinar las diferencias estadísticamente significativas entre especies, técnicas y en la interacción técnica-especie para cada caso. El contraste de curvas de supervivencia se realizó mediante modelos no paramétricos de Kapplan-Meier (Fox, 2001) con el programa JMP 8.0.2.

Las variables de diámetro y altura se analizaron mediante la fórmula de tasa de crecimiento relativo (TCR) (Hunt, 1978):

$$
T C R=\frac{\ln \left(h_{2}\right)-\ln \left(h_{1}\right)}{t_{2}-t_{1}}
$$

donde $\mathrm{h}$ es altura $(\mathrm{cm})$ o diámetro $(\mathrm{mm})$ y $t_{1}$ y $t_{2}$ son tiempo inicial y final en meses, respectivamente.

\section{Resultados}

\section{Supervivencia y estado de las plantas}

La supervivencia fue de $53.8 \%$ con la técnica de translocación y 58.4\% de producción en vivero (independientemente a la especie). La tendencia de la supervivencia para cada especie fue similar entre translocación y plantas de vivero: mayores porcentajes de Ocotea puberula (80\% - 82\%, respectivamente), seguida de Beilschmiedia anay (46\% - 61\%) y Pseudolmedia glabrata (33\% 31\%) (Fig. 3).

La supervivencia fue significativamente diferente entre especies tanto con la técnica de translocación (Wilcoxon: $\left.X^{2}=23, \mathrm{p}<0.0001\right)$ como para los individuos provenientes de vivero (Wilcoxon: $\mathrm{X} 2=24.12, \mathrm{P}<0.0001$ ); en ambos casos la secuencia de mayor a menor fue Ocotea puberula, Beilschmiedia anay y Pseudolmedia glabrata. En relación con las potenciales discrepancias de cada una de las especies, en función de la técnica de establecimiento, se observó que en O. puberula no hay diferencias estadísticamente significativas (Wilcoxon: $\left.X^{2}=0.03, \mathrm{p}=0.85\right) ; B$. anay presentó diferencias marginalmente significativas (Wilcoxon: $X^{2}=$ 3.31, $\mathrm{p}=0.068$ ), pues casi todo el año fue más alta la supervivencia para la técnica de producción en vivero. Sin embargo, las diferencias en supervivencia entre las técnicas fueron compensadas. P. glabrata no mostró diferencias estadísticamente significativas entre las técnicas (Wilcoxon: $\left.X^{2}=0.051, \mathrm{p}=0.82\right)$ (Fig. 3).

\section{Estado fisiológico de los individuos por especie}

\section{Ocotea puberula}

Bajo la técnica de translocación, esta especie tuvo los mayores porcentajes de individuos vivos durante los primeros cinco meses; sin embargo, al cabo de 13 meses la técnica de producción en vivero presentó $40 \%$ de individuos vivos. De la misma forma, la técnica de translocación tiene los porcentajes más bajos de estrés durante los primeros cinco meses, pero en el mes 13 alcanza $56.84 \%$. En relación con la mortalidad de individuos, el porcentaje más alto se registró en diciembre $(35.79 \%)$ con la técnica de producción en vivero; sin embargo, en el mes trece, el porcentaje de individuos muertos cayó a 18.95\%; esto significa que durante ocho meses hubo $16.84 \%$ de retoños. Con la técnica de translocación, el valor más alto de mortalidad al cabo de trece meses fue de 20\% (Fig. 4). 


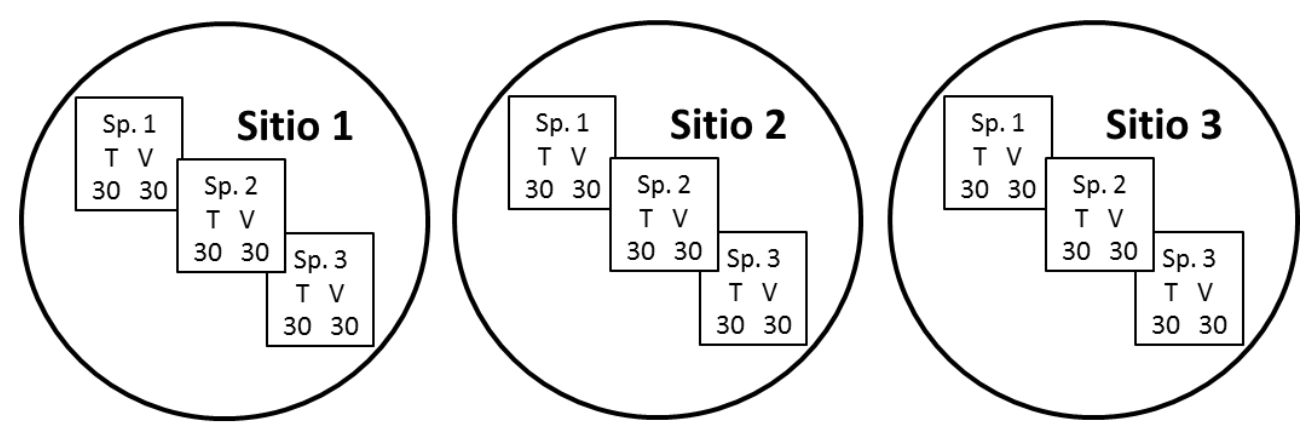

FIGURA 2. Diseño, distribución y número de plantas por sitio para ensayo de enriquecimiento.

Sp.1: Beilschmiedia anay, Sp.2: Ocotea puberula, Sp.3: Pseudolmedia glabrata, T: translocación, V: producción en vivero

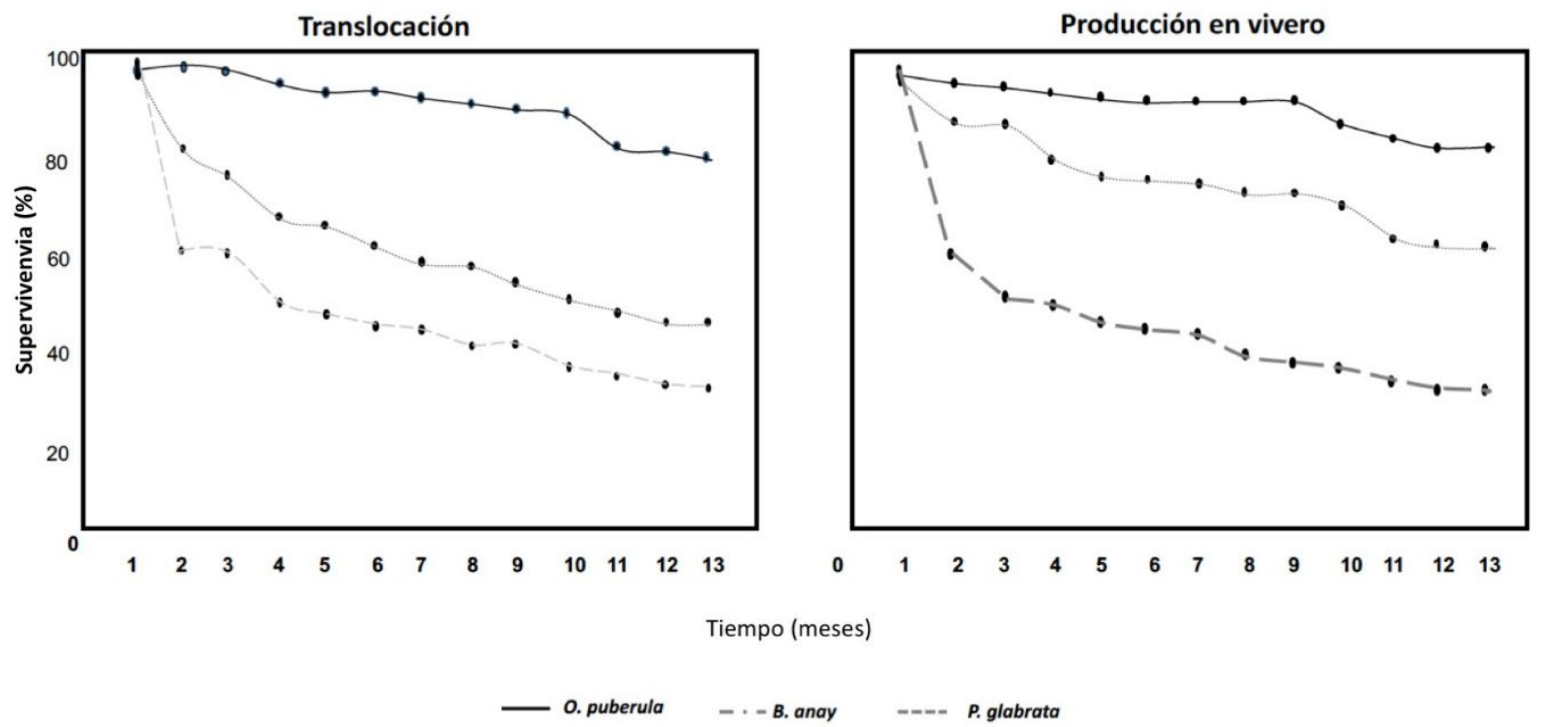

FIGURA 3. Porcentaje de supervivencia de tres especies en las técnicas de translocación y producción en vivero.

\section{Beilschmiedia anay}

El porcentaje de individuos vivos fue más alto con la técnica de translocación durante los primeros cinco meses; en cambio, del sexto en adelante la técnica de producción en vivero presentó los valores más altos, hasta $32.99 \%$ en el último mes. Los valores más altos de estrés para esta especie se presentaron con la producción en vivero, entre $6 \%$ en el mes 10 y $70 \%$ en el mes 1 . Contrario a este caso, el mayor porcentaje de estrés con la técnica de translocación, se registró en el mes 12 con 53.61\%, lo que sugiere que, mientras los individuos producidos en vivero tuvieron estrés post-manipulación, los individuos translocados se estresaron en temporada de lluvias. Otro factor de estrés que afectó las plántulas de $B$. anay durante los primeros cinco meses fue el intento de mamíferos pequeños por comer la semilla. La translocación presentó $53.61 \%$ de las plántulas muertas durante el último mes de monitoreo; la producción en vivero $55.67 \%$ en el quinto mes y $39.18 \%$ en el último mes; es decir, en ocho meses hubo $16.49 \%$ de retoños (Fig. 4). 

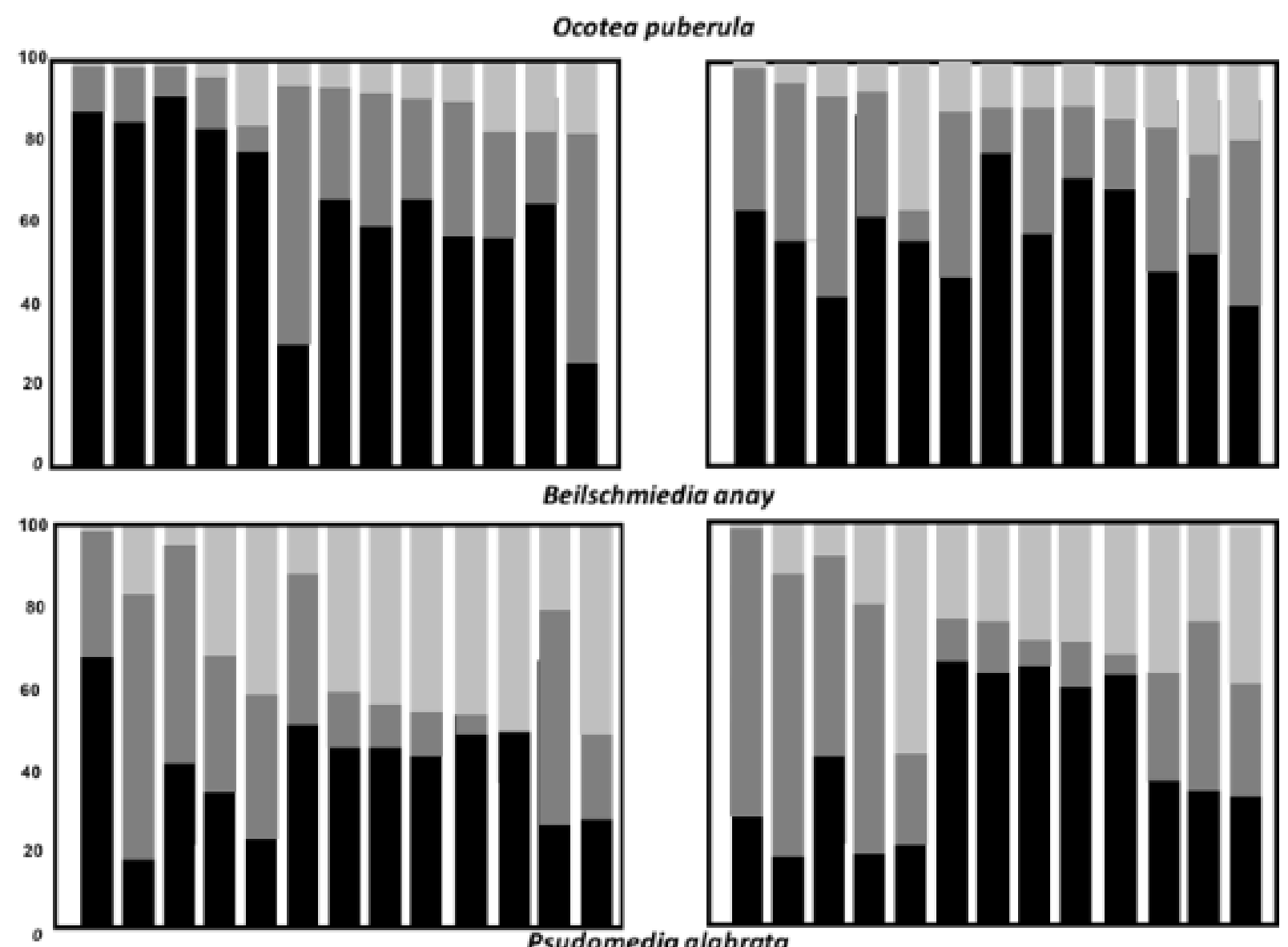

miedia anay
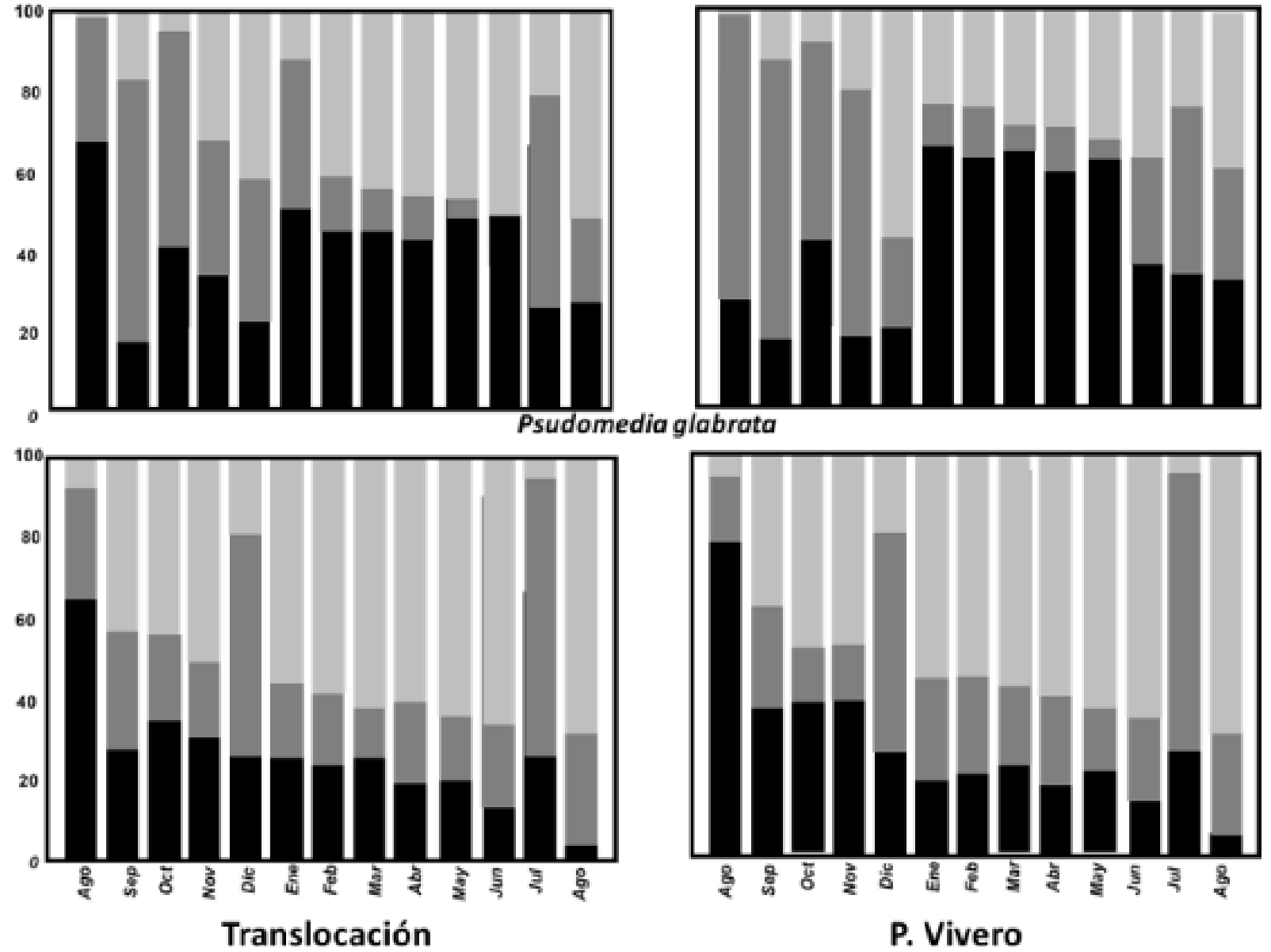

$\%$ Vivas

$\%$ Estresadas

\% Muertas

FIGURA 4. Porcentajes de plántulas vivas, muertas y estresadas de a) Ocotea puberula, b) Beilschmiedia anay y c) Pseudolmedia glabrata. 


\section{Pseudolmedia glabrata}

El mayor porcentaje de plántulas vivas $(6.59 \%)$ se dio mediante la aplicación de la técnica de producción en vivero. Con la translocación se presentó, en el tercer mes, el porcentaje más alto para individuos estresados (27.47\%). Con ambas técnicas se registró el porcentaje más alto de estrés (68.13\%) en el mes 12 (julio) y entre el mes 11 y 12 , $60.44 \%$ de retoños. El porcentaje de individuos muertos en el mes 13 fue de $69.23 \%$ para las dos técnicas, lo que se puede relacionar con el intenso periodo de lluvias que se registró en agosto de 2013 (Fig. 4).

\section{Crecimiento de plántulas}

El análisis de varianza para TCR en altura durante un año mostró diferencias estadísticamente significativas entre especies ( $p=0.000673)$; de igual manera, en la interacción especie-técnica resultó significativa $(\mathrm{F}=3.9048, \mathrm{p}=$ 0.02142). La comparación de medias por Tuckey reveló que
Ocotea puberula es la especie con diferencias estadísticamente significativas en TCR en altura entre técnicas, alcanzando $0.26 \mathrm{~cm}$ de TCR en translocación (Fig.5).

$\mathrm{El}$ análisis de varianza para TCR en diámetro de las tres especies al final de 11 meses demostró que hubo diferencias estadísticamente significativas entre especies $(p<0.05)$ y en la interacción especie-técnica $(F=6.0425, \mathrm{p}=0.00274)$ (Fig. 6). La comparación de medias de Duncan indicó que la especie Psendolmedia glabrata fue la única especie con diferencias estadísticamente significativas en TCR en diámetro. (Fig. 6). Se observó también que Beilschmiedia anay y P. glabrata alcanzaron su mayor crecimiento diamétrico, con medias de 0.09 y 0.25 respectivamente, mediante la producción en vivero. Ocotea puberula tuvo mayor crecimiento con la técnica de translocación, una media de 0.16. La tendencia de crecimiento en diámetro para cada técnica fue la misma, en ambos crece más $P$. glabrata, seguida de O. puberula y B. anay.

\section{Crecimiento altura 0.95 intervalo de confianza}

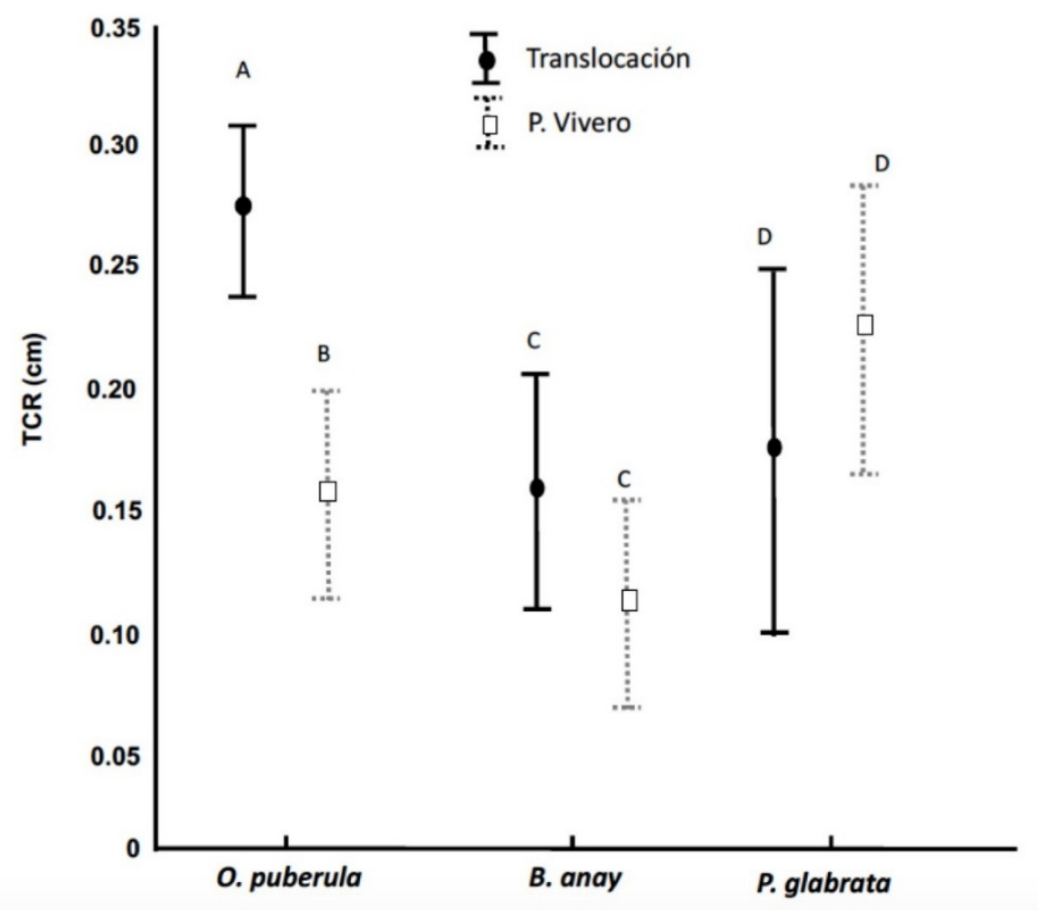

FIGURA 5. Comparación de la tasa de crecimiento relativo a los 12 meses para altura de Ocotea puberula, Beilschmiedia anay y Pseudolmedia glabrata bajo las dos técnicas de enriquecimiento. 


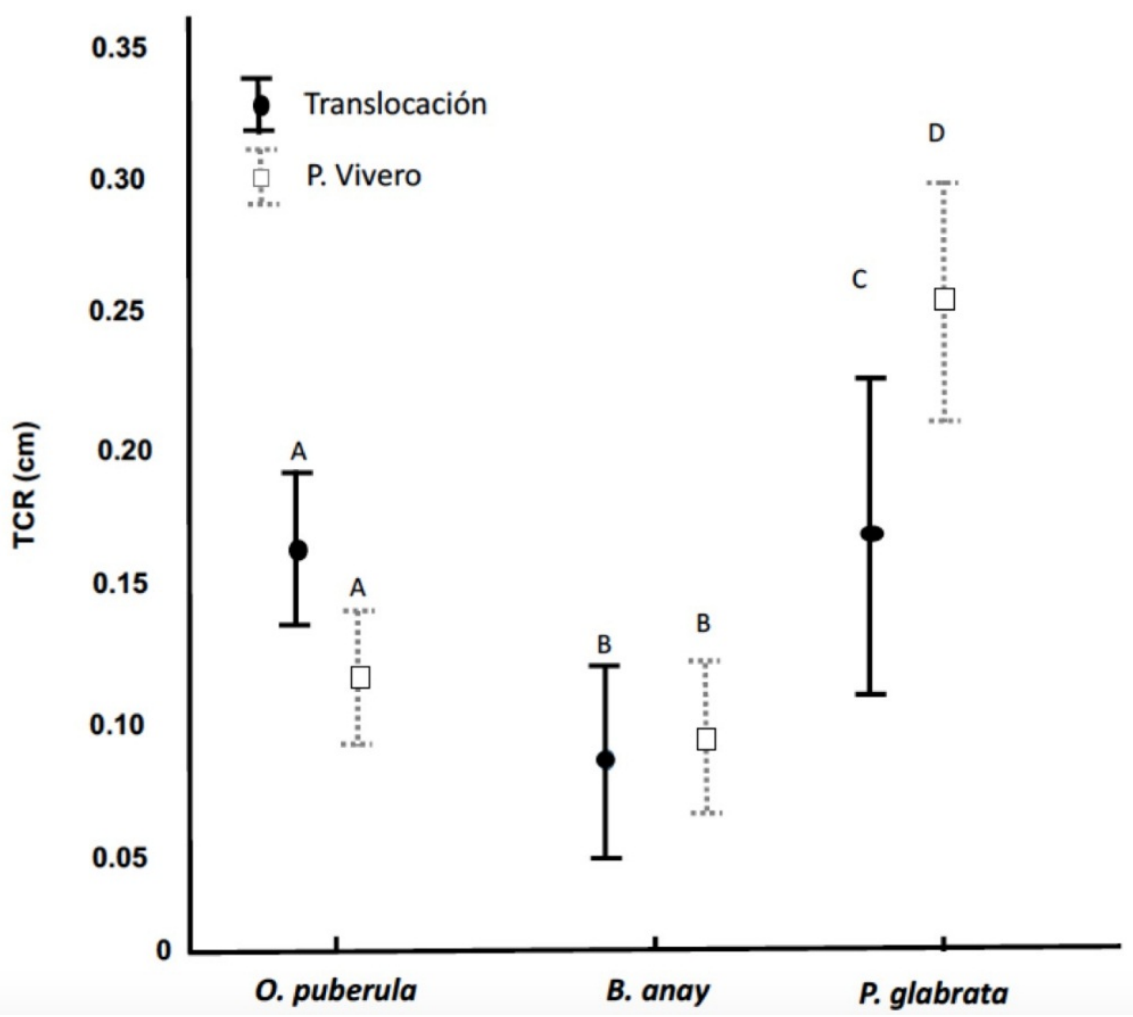

FiguRA 6. Comparación de la tasa de crecimiento relativo de 11 meses para diámetro de Ocotea puberula, Beilschmiedia anay y Pseudolmedia glabrata en dos técnicas de enriquecimiento.

Comparación de medias de Duncan (A, B, C y D), letras iguales no difieren significativamente ( $>$ > 0.05).

\section{DISCUSIÓN}

En este trabajo se muestra el potencial de la técnica de translocación y plantas de vivero para el enriquecimiento de un bosque oligárquico de origen antrópico. En general se observó una alta supervivencia, superior a 50\% para las plantas de las tres especies, con las dos técnicas de siembra. En el caso de Ocotea puberula se encontraron valores altos (arriba de 80\%) e indistinguibles entre las técnicas. Estos valores de supervivencia son comparables a los obtenidos con Quercus acutifolia (80\%) registrados por Alvarez-Aquino et al. (2004) en un bosque mesófilo de montaña, aunque en su caso las plantas provenían de vivero. Por otro lado, Beilschmiedia anay presentó 15\% mayor supervivencia con la técnica de vivero, mientras que Psendolmedia glabrata, es ligeramente mayor por la vía de la translocación, aunque para ninguna de las tres especies se registró diferencia estadísticamente significativa con respecto a las técnicas empleadas. Es decir, para las tres especies fue indistinto usar una técnica u otra para enriquecer los cachichinales.

En términos del cambio en el estado fisiológico que presentaron las plantas a lo largo de los 13 meses del estudio, se observaron niveles de estrés considerables para todas las especies durante la primera etapa de su establecimiento, aunque, para Psendolmedia glabrata, se muestra un alto estrés hacia el penúltimo mes con las dos técnicas de siembra (Fig. 4). Es bien conocido que las plántulas recién plantadas tienen una baja permeabilidad del sistema radicular y/o un contacto pobre en la interface raízsuelo que limita la absorción de agua y nutrientes, por lo que las plántulas expresan estrés justo después de la siembra 
(Grossnickle, 2005). Mediante la translocación el riesgo de pérdida de raíces es alto y las plantas dependen del tamaño del sistema radicular mantenido durante el proceso; esto determina la capacidad de las plántulas de absorber el agua para iniciar el establecimiento (Carlson, 1986). Sin embargo, la técnica empleada correctamente en plántulas con un volumen de raíz más grande, aumenta la capacidad de absorción de agua y evita (o al menos disminuye) el estrés de siembra y por lo tanto, hay mayor supervivencia. Es importante tomar en cuenta las prácticas adecuadas de silvicultura (cuidado en vivero, extracción cuidadosa y manejo de raíces en el caso de la translocación), en combinación con plántulas en buen estado fisiológico (sin plagas, enfermedades y efectos por herbivoría). La siembra de las plantas durante el período de lluvias, aumenta la posibilidad de desarrollar raíces en el sistema del suelo circundante y establecerse exitosamente (Grossnickle, 2005).

En cuanto al crecimiento, se encontró que Ocotea puberula muestra mayores valores cuando es introducida mediante la técnica de translocación, esto fue evidente tanto en diámetro como en altura. En términos de crecimiento, O. puberula puede ser recomendada para el enriquecimiento de la zona y obtener individuos más grandes en menor tiempo, debido a una tasa de crecimiento más alta para esta especie. Un estudio en Misiones, Argentina, dirigido a generar información ecológica de 11 especies para el enriquecimiento de bosques secundarios, concluye que en siete años $O$. puberula tiene altos valores de crecimiento en altura $(1.79 \mathrm{~m}-4.11 \mathrm{~m})$ y diámetro $(5.49 \mathrm{~cm}-6.05 \mathrm{~cm})$; además de importantes porcentajes de supervivencia ( $40 \%$ - 75\%), por tal motivo, es considerada como una especie apta para el enriquecimiento de bosques (Montagnini et al., 1997), lo anterior, coincide con los resultados de este trabajo. Además, a escala local, es importante por el valor comercial de su madera y la baja susceptibilidad a ser invadida por plagas. Al considerar que O. puberula se encuentra en la lista roja de la International Union for Conservation of Nature's Red List of Threatened Species [IUCN]
2018) bajo la categoría de "bajo riesgo", su incursión en los cachichinales puede ayudar a disminuir la presión sobre las poblaciones naturales.

Estos resultados tienen dos vertientes alentadoras: la primera se refiere a la alta capacidad de supervivencia de las tres especies $(30 \%-80 \%)$, valores similares a los registrados por Alvarez-Aquino, Williams-Linera y Newton (2004) en las plantaciones forestales (alrededor de 60\%) y en la restauración en bosque mesófilo (15\% - 50\%). En segundo lugar, la translocación es un método que no difiere en su efectividad al compararlo con la técnica de manejo de vivero. Esto implica un menor costo si se compara con los generados en un vivero como son la recolecta, el beneficiado de semillas, construcción, el mantenimiento de plantas, así como el traslado a los sitios de siembra, entre otros aspectos. Es recomendable la extracción de plántulas cercanas a los árboles madre con el fin de ser aprovechadas, ya que al permanecer allí, la probabilidad de muerte por patógenos, herbívoros y/o competencia es alta, tal y como lo predice la hipótesis de Janzen (1970) y Connell (1971), por lo que esta extracción no impactaría a las poblaciones naturales.

\section{CONCLUSIONES}

Los ensayos de este estudio demuestran la factibilidad técnica de incorporar especies de valor comercial y cultural a un bosque oligárquico de origen antrópico, como es el cachichinal. Se concluye que las dos técnicas utilizadas han sido evaluadas positivamente y pueden ser aplicadas indistintamente para la introducción de plantas nativas en los cachichinales, las tres especies seleccionadas presentaron una alta supervivencia y crecimiento. El enriquecimiento implica una acción de doble propósito: por un lado, mejorar el funcionamiento y valor ecológico de los cachichinales (mayor riqueza y cobertura) y por el otro, procurar el bienestar de las comunidades para proveer de recursos que impulsen el valor económico de sus bosques al aumentar la diversidad de las especies. 


\section{RECONOCIMIENTOS}

Se agradece de manera especial a los habitantes de Pueblo Viejo, Mpio. Misantla, Veracruz por las facilidades para llevar a cabo este estudio, en especial a la familia López Lagunes. La primera autora agradece el apoyo del Consejo Nacional de Ciencia y Tecnología por haber recibido la beca 444324 en el programa 003057 de la Maestría en Ciencias en Ecología Forestal del Instituto de Investigaciones Forestales de la Universidad Veracruzana. Los autores agradecen a Reyna Paula Zárate por la revisión de estilo y formato del manuscrito y las referencias, a José Luis Álvarez por la elaboración del mapa de ubicación de la zona de estudio y a Gregoria Zamora por la edición de las figuras.

\section{REFERENCIAS}

Aide, T. M., Zimmerman, J. K., Pascarella, J. B., Rivera, L., \& Marcano-Vega, H. (2000). Forest regeneration in a chronosequence of tropical abandoned pastures: implications for restoration ecology. Restoration ecology, 8(4): 328-338. doi: 10.1046/j.1526-100x.2000.80048.x

Alvarez-Aquino, C., Williams-Linera, G., \& Newton, A. C. (2004). Experimental native tree seedling establishment for the restoration of a Mexican cloud forest. Restoration Ecology, 12(3), 412-418.

Carlson, W. C. (1986). Root system considerations in the quality of loblolly pine seedlings. Southern Journal of Applied Forestry, 10(2), 87-92.

Connell, J. H. (1971). On the role of natural enemies in preventing competitive exclusion in some marine animals and in rain forest trees. En P. J. den Boer, \& G. R. Gradwell. Dynamics of populations (pp. 298-310). Wageningen, The Netherlands: Centre for Agricultural Publications and Documentation.

Covarrubias, M. (2010). Estructura y diversidad vegetal asociada a la presencia de Oecopetalum mexicanum Greenm. \& C.H. Thomps., (Icacinaceae) en tres sistemas de manejo de la sierra de Misantla, Veracruz, México. Tesis de licenciatura no publicada. Universidad Veracruzana, Facultad de Biología, Xalapa, Veracruz, México.

Fox, G. A. (2001). Failure-time analysis. En S. M. Scheiner, \& J. Gurevitch (Eds.). Design and analysis of ecological experiments (pp. 235-266). EUA: Oxford University Press.

Gómez-Pompa, A. (1966). Estudios botánicos en la región de Misantla, Veracruz. México, D.F., Imernar.
Grossnickle, S. C. (2005). Importance of root growth in overcoming planting stress. New Forests, 30(2-3), 273-294.

Gutiérrez, B. C. (1993). Listado florístico de la Sierra de Chiconquiaco, Ver. Textos Universitarios. Xalapa, Veracruz: Universidad Veracruzana.

Gutiérrez, C. L., \& Dorantes L, J. (2007). Especies forestales de uso tradicional del estado de Veracruz. México: Conafor-ConacytUV.

Hunt, R. (1978). Plant growth analysis. Serie: Studies in Biology, no. 96. Londres, Inglaterra: Edward Arnold (publishers) Ltd.

Janzen, D. H. (1970). Herbivores and the number of tree species in tropical forests. American Naturalist, 104(940), 501-528.

Lascurain, M., Avendaño-Reyes, S., López-Binnqüist, C., LópezAcosta, J. C., Covarrubias-Báez, M., \& Duno-de Stefano, R. (2013). Uso y flora leñosa asociada a Oecopetalum mexicanum (Icacinaceae): una especie comestible nativa de la sierra de Misantla, Veracruz, México. Botanical Sciences, 91(4), 477-484. doi: 10.17129/botsci.424

Lascurain, M, López-Binnqüist, C, \& Emery, M. (2016). Culture and environment in the Sierra de Misantla, Veracruz, Mexico: the case of Oecopetalum mexicanum. Madera y Bosques, 22(3), 11-21. doi: 10.21829/myb.2016.223489

López-Acosta, J. C., Lascurain, M., López-Binnqüist, C., \& Covarrubias, M. (2014). Structure and floristic composition of forest management systems associated with the edible fruit tree Oecopetalum mexicanum in the Sierra de Misantla, Veracruz, Mexico. Economic Botany, 68, 44-58. doi: 10.1007/s12231-014-9260-0

Mansourian, S., Lamb, D., \& Gilmour, D. (2005). Overview of technical approaches to restoring tree cover at the site level. In S. Mansourian, D. Vallauri, \& N. Dudley (Eds.). Forest restoration in landscapes (pp. 241-249). New York, NY: Springer.

Martínez-Garza, C., \& Howe, H. F. (2003). Restoring tropical diversity: beating the time tax on species loss. Journal of Applied Ecology, 40(3), 423-429.

Michon, G. (2005). Domesticating forests: how farmers manage forest resources. Jakarta, Indonesia: Institut de Recherche pour le Développement-Center for International Forestry Research-The World Agroforestry Centre.

Montagnini, F., Eibl, B., Grance, L., Maiocco, D., \& Nozzi, D. (1997). Enrichment planting in overexploited subtropical forest of the Paranaense region of Misiones, Argentina. Forestry Ecology and Management, 99(1-2), 237-246. 
Niembro-Rocas, A., Morato, I., \& Cuevas-Sánchez, J. A. (2004) [CD-ROM]. Catálogo de frutos y semillas de árboles y arbustos de valor actual y potencial para el desarrollo forestal de Veracruz y Puebla. (Reporte final del Proyecto Conacyt-Conafor2002-CO1-5741). Xalapa, México: Instituto de Ecología, A.C.

Paquette, G., Léonard, M., Lundgren-Cayrol, K., Mihaila, S., \& Gareau, D. (2006). Learning design based on graphical knowledge-modelling. Educational Technology \& Society, 9(1), 97-112.

Pennington, T. D., \& Sarukhán, J. (2005). Árboles tropicales de México. Manual para la identificación de las principales especies. México: Fondo de Cultura Económica.

Peters, C. M. (2000). Precolumbian silviculture and indigenous management of Neotropical forests. En D. L. Lentz (Ed.), Imperfect Balance: Landscape Transformations in the PreColumbian Americas (pp. 203-224). Nueva York: Columbia University Press.

Schroth, G., da Fonseca, G. A. B., Harvey C. A., Gascon, C., Vasconcelos, H. L. e Izac, A. M. N. (2004). Agroforestry and biodiversity conservation in tropical landscapes. Washington, D.C.: Island Press.

Ticktin, T., Johns, T., \& Xoca, V. C. (2003). Patterns of growth in Aechmea magdalenae (Bromeliaceae) and its potential as a forest crop and conservation strategy. Agriculture, Ecosystems \& Environment, 94(2), 123-139.

Union for Conservation of Nature's [IUCN] (2018). Red List of Threatened Species. Recuperado de http:/ /www.iucnredlist.org/about.

Manuscrito recibido el 12de julio de 2017

Aceptado el 25 de abril de 2018

Publicado el 26 de noviembre de 2018

Este documento se debe citar como:

Covarrubias, M., López-Acosta, J. C., Lascurain-Rangel, M., Rebolledo, V., Pedraza, R. A., \& Avendaño-Reyes, S. (2018). Bosques oligárquicos de Oecopetalum mexicanum enriquecidos con especies nativas de la Sierra de Misantla, México. Madera y Bosques, 24(3), e2431596. doi: 10.21829/myb.2018.2431596

Madera y Bosques por Instituto de Ecología, A.C. se distribuye bajo una Licencia Creative Commons Atribución-NoComercialCompartirlgual 4.0 Internacional. 